\title{
Experimental Measurements on the Thermal Conductivity of Glycerol-Based Nanofluids with Different Thermal Contrasts
}

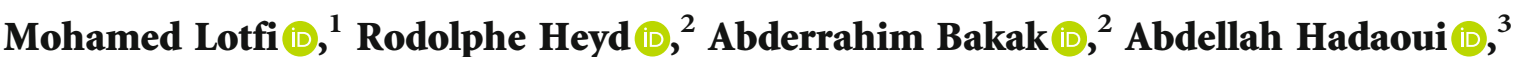 \\ and Abdelaziz Koumina $\mathbb{B D}^{2}$ \\ ${ }^{1}$ LaMEE, Cadi Ayyad University, FSSM, B.P. 2390, Marrakech 40000, Morocco \\ ${ }^{2}$ LIRBEM, Cadi Ayyad University, ENS, Route d'Essaouira, Marrakech 40000, Morocco \\ ${ }^{3}$ Radiotherapy Department, Regional Center of Oncology, Agadir 80000, Morocco
}

Correspondence should be addressed to Mohamed Lotfi; lotfimohamed_1999@yahoo.fr

Received 25 April 2021; Revised 12 August 2021; Accepted 18 August 2021; Published 8 September 2021

Academic Editor: Donglu Shi

Copyright (c) 2021 Mohamed Lotfi et al. This is an open access article distributed under the Creative Commons Attribution License, which permits unrestricted use, distribution, and reproduction in any medium, provided the original work is properly cited.

\begin{abstract}
We report, in this work, our study of the thermal conductivity of high-viscosity nanofluids based on glycerol. Three nanofluids have been prepared with different thermal contrasts, by suspending graphene flakes, copper oxides, or silica nanoparticles in pure glycerol. The nanofluids were thermally characterized at room temperature with the $3 \omega$ technique, with low amplitudes of the temperature oscillations. A significant enhancement of the thermal conductivity is found in both the glycerol/copper oxide and the glycerol/graphene flake nanofluids. Our results question the role played by the Brownian motion in the microscopic mechanisms of the thermal conductivity of high-viscosity glycerol-based nanofluids. A similar behavior of the thermal conductivity as a function of the nanoparticle volume fraction was found for all three glycerol-based nanofluids presently investigated. These results could be explained on the basis of fractal aggregation in the nanofluids.
\end{abstract}

\section{Introduction}

Over the last few years, the improvement of thermal transfer has become an imperative requirement for optimizing the use of energy in various industrial and technological fields, mainly due to the scarcity of fossil fuels exhorting the use of current resources more efficiently. Among the different venues explored in the past two decades to improve the transfer of heat, the use of nanofluids has become an attractive alternative that has been intensively studied by the international scientific community. According to the terminology introduced by Choi in 1995 [1], a nanofluid consists of a stable dispersion of nanoparticles (NPs) in a base liquid. This kind of dispersion has aroused considerable interest in the last few years since a significant improvement of the effective thermal conductivity has been achieved with several nanofluids. It has been demonstrated that the dispersal of highly conductive nanoparticles in liquids, even in very small amounts (less than $1 \%$ per volume), is able to increase the effective thermal conductivity of the base fluid by nearly $200 \%$ with carbon nanotubes (CNTs) for example [2] and by about $40 \%$ in the case of the copper oxide NPs [3]. To retain the configuration and volume of a heat dissipation system (e.g., a battery-cooling system in the constraint trunk of an electric vehicle) and to raise heat dissipation capacity, nanofluids can be used because they transfer heat efficiently [4]. For example, with regard to the use of CNTs in heat dissipation, the heat transfer performance of CNT/water nanofluids (CNWNFs) was measured [5]; the measurements revealed that using CNTs at a concentration of $0.5 \mathrm{wt} \%$ in a horizontal tube enhanced heat transfer by $350 \%$.

In spite of these encouraging results, questions remain about the interest of these fluids concerning heat transfer. These doubts originate from the high variability of the experimental results reported to date in the literature, e.g., the reviews of Wang and Mujumdar [6] and Aybar et al. [7]. As mentioned by Corcione [8], the discrepancies among the data available in the literature are sometimes of the order 
of $50 \%$ or more. For a given type of NPs, the reasons for these discrepancies vary and may originate from differences in the sample preparation (NP synthesis modes, size, shape, oxidation, dispersion modes, chemical additives used and agglomeration, and base liquids used) or in the measurement methods (transient hot wire, transient plane source, $3 \omega$ method, and hot disk). Among all these reasons, the state of dispersion and agglomeration of the NPs undoubtedly plays a key role.

Several studies $[9,10]$ have shown that the thermal conductivity of water and ethylene glycol-based nanofluids decreases dramatically over time, returning to the value of the base fluid in about ten minutes after the dispersal. This time-dependent behavior of the thermal conductivity is due to particle agglomeration and consequently to settling.

To avoid this harmful influence of clustering, some chemical additives are commonly used to ensure a longer stability of the solution. Control of the $\mathrm{pH}$ value, use of surfactants, and polymeric grafting at the surface of the NPs are common stabilizing methods against settling. Unfortunately, these surface treatments may alter the way in which heat is transferred inside the suspension and, thus, hide the real influence of the NPs on the effective thermal conductivity of the nanofluid [11]. Therefore, the use of various stabilization routes and chemical additives, different from one experimental study to another, can contribute to the variations of the experimental results. In addition, the use of base liquids with low viscosities, such as water $(\eta=1 \mathrm{mPa} \cdot \mathrm{s}$ at room temperature), inevitably questions the influence of convection on the measurement of the thermal conductivity, both at the microscopic and macroscopic levels.

This confusion is also reflected in the large number of models proposed to explain the various experimental results $[6,7]$. Some authors [10] have even shown that at low concentrations (less than 1\%), no significant improvement in conductivity is observed in the case of well-dispersed nanofluids, for which the results of effective-medium theories, such as the Maxwell-Garnett and Hamilton-Crosser laws, still apply. Using nanofluids containing magnetite nanoparticles [12-14], Philip et al. have shown that initially welldispersed nanoparticles do not induce significant thermal conductivity (TC) enhancement of their suspensions. In contrast, when applying a magnetic field to their suspensions, they have obtained significant enhancements of the TC, by inducing linear agglomerates of the magnetite nanoparticles in the base fluids. They have shown that the observed enhancements of the TC were due to the effective heat transport through the induced chainlike aggregates of nanoparticles, rather than to the Brownian microconvection.

In order to limit the influence of both chemical treatments and convection, our suspensions were prepared with a high-viscosity base liquid. This choice can help to identify as clearly as possible the real thermal diffusive properties of the nanofluid.

Glycerol was chosen as the base fluid, due on the one hand to its high viscosity at room temperature and to the lack of specific reactivity with the NPs used here. Compared to water, glycerol has several advantages in the framework of our study. Its high viscosity allows us to obtain stable sus- pensions without any specific functionalization and without using chemical additives for promoting the dispersion. As mentioned earlier, this chemical neutrality is highly important. It allows us to, macroscopically, characterize the effective thermal conductivity of the nanofluid, without the microscopic influence of chemical treatments, usually unavoidable with less viscous fluids. In addition, as mentioned by Moghaddam et al. [15], glycerol has the correct dispersion solubility needed to prepare stable graphene suspensions.

Many researchers have observed that the thermal conductivity enhancement along with rising temperature, regardless of the selection of nanomaterials, is due to the Brownian motion, where absorption of kinetic energy causes more particle collisions. However, the nanofluid's thermal performance generally would depend on the appropriate amount of concentrations of weight/volume percentages, which will jeopardize others [16]. One of the main objectives of this work is to analyze the influences of the Brownian microconvection and aggregation on the enhancement of the thermal conductivity of highly viscous liquids, as a function of the solid volume fraction.

\section{Experimental Methods}

2.1. Preparation of the Samples. In the present study, NPs showing different thermal conductivities $k$ have been used, regardless of their sizes and shapes. Silica spherical particles (Figure 1(a)), with $50 \mathrm{~nm}$ average diameters, were synthesized in the laboratory by using the Stöber process [17]. They correspond to the lowest thermal conductivity studied here. Copper oxide NPs, with $7 \mathrm{~nm}$ average diameters (Figure 1(b)), were synthesized in the laboratory by the thermal decomposition of copper (II) acetylacetonate with oleylamine [18]. The process has been improved by the addition of a small quantity of trioctylamine during the decomposition [19]. Due to the very high reactivity of these small $\mathrm{NPs}$, the resulting powder is made of a mixing of $\mathrm{CuO}$ and $\mathrm{Cu}_{2} \mathrm{O}$ particles, as revealed by XPS analysis performed on these copper oxide NPs just before the introduction in the glycerol.

Finally, we also have used a graphene nanopowder, composed of flakes with $8 \mathrm{~nm}$ thickness, $550 \mathrm{~nm}$ average lateral size, specific surface area of $100 \mathrm{~m}^{2} / \mathrm{g}$, and purity $>99.9 \%$. It has been bought from the Graphene Supermarket Company and dispersed as received in glycerol, without any other chemical treatment. Figure 2 shows the scanning electron microscope (SEM) images of the GNP sheets. Graphene NPs are supposed to exhibit the highest thermal conductivity tested in the present study.

Nanoparticles were chosen with varied thermal conductivities:

(i) Graphene has an outstanding thermal conductivity. It is thus expected that the use of graphene-based nanofluids can significantly enhance thermal characteristics of heat transfer fluids (HTFs)

(ii) Graphene-based nanofluids have lower environmental impacts 


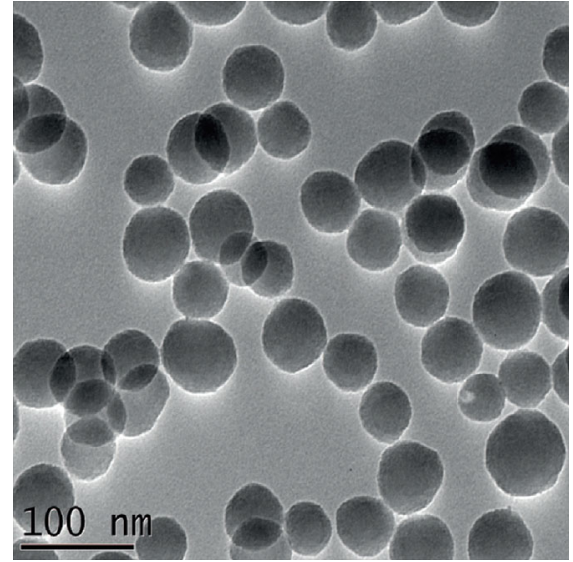

(a)

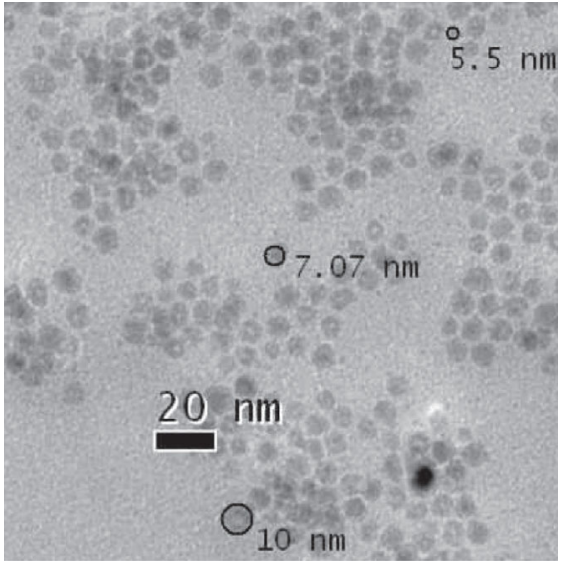

(b)

Figure 1: Transmission electron microscope (Philips CM20 TEM) pictures of the different types of nanoparticles used in this study. (a) $\mathrm{SiO}_{2}$ NPs with $50 \mathrm{~nm}$ average diameter, synthesized by using the Stöber process. (b) Copper oxide NPs with 7 nm average diameter, synthesized by a thermal decomposition process. The NPs were synthesized at the ICMN institute.
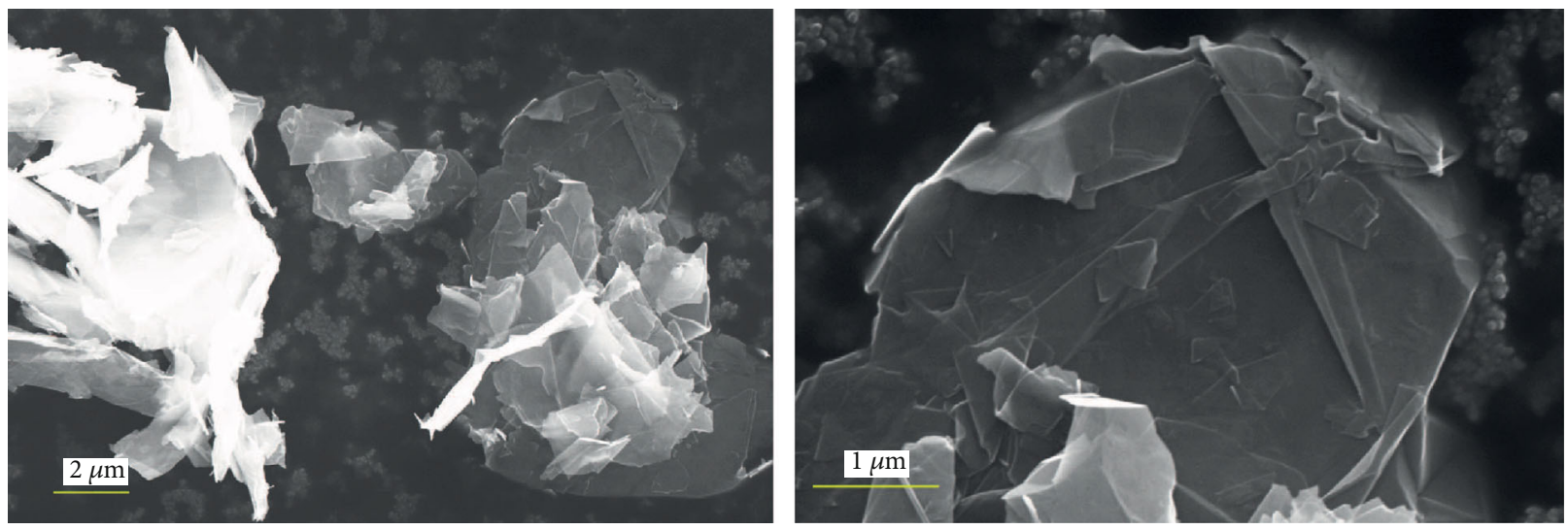

Figure 2: SEM images of GNP nanosheets (Zeiss Supra 25 microscope with EDS probe and $1.5 \mathrm{~nm}$ resolution at $20 \mathrm{kV}$ ).

(iii) $\mathrm{CuO}$ and $\mathrm{SiO}_{2}$ nanoparticles exhibit different thermal conductivity contrasts

Because of the high hygroscopicity of glycerol, only fresh liquid (Sigma-Aldrich, glycerol for molecular biology $\geq 99 \%$ ) has been used to prepare the suspensions. Hermetic precautions, concerning the storage of the samples and the operation of the measurement cell, were implemented to avoid the dissolution of the atmospheric water vapor in the nanofluid. To enable a good homogenization of the suspension at the macroscopic scale, the NPs are progressively introduced into heated glycerol (at a temperature of $50^{\circ} \mathrm{C}$ ), in order to decrease its viscosity and ease the homogenization process. A volume of $20 \mathrm{~mL}$ of this moderately heated suspension is then stirred and finally sonicated at low power $(160 \mathrm{~W}-35 \mathrm{kHz})$ during 48 hours in order to break the aggregates. The more concentrated suspensions were prepared first using an analytical balance $(0.0001 \mathrm{~g})$, to precisely measure the mass of the NPs. The lower volume fraction suspensions were prepared by dilution of the more concentrated, by adding the required amounts of pure glycerol.
The values of the different thermal conductivities of the materials used in this study are summarized in Table 1 (room temperature).

2.2. Suspension Characterization. To study the state of dispersion of the nanoparticles in the base fluid and in order to evaluate the presence and size of graphene aggregates, samples of each suspension were analyzed by SEM after drying. Figure 3 shows an example of nanofluid based on glycerol, for a volume fraction $0.25 \%$ of graphene. One drop of each sample was collected, placed on the SEM grid and then slowly dried in an oven. Figure 3 shows that the graphene nanosheets are uniformly dispersed and that the morphology of these nanosheets is not altered after the stirring and sonication steps.

\subsection{Experimental Setup for the Thermal Characterization}

2.3.1. Mode of Operation. In order to measure the effective thermal conductivity of our suspensions, we have adapted the $3 \omega$ technique, developed by Cahill for the thermal characterization of dielectric solids [20], to the case of electrically 
TABLE 1: Typical values of the materials thermal conductivities used in this study.

\begin{tabular}{lcccc}
\hline Materials & Glycerol & $\begin{array}{c}\text { Silica } \\
\text { NPs }\end{array}$ & $\begin{array}{c}\text { Copper oxide } \\
\text { NPs }\end{array}$ & $\begin{array}{c}\text { Graphene } \\
\text { flakes }\end{array}$ \\
\hline$k\left(\mathrm{~W} \cdot \mathrm{m}^{-1} \cdot \mathrm{K}^{-1}\right)$ & 0.28 & 1.4 & 50 & $1.5 \times 10^{3}$ \\
\hline
\end{tabular}

conducting liquids [21,22]. This method has been privileged here, rather than the usual transient hot wire (THW) technique, because the use of a very efficient dual-phase synchronous detector (DPSD) allows to use very low amplitudes of the sensing wire temperature (of the order of $1 \mathrm{~K}$ ), which reduces the influence of natural convection on the thermal diffusivity measurement.

The measurement temperature $T_{i}$ of the nanofluid was stabilized using a thermostatic bath $(0.1 \mathrm{~K})$. A low amplitude excitation current $i(t)=\widehat{I} \cos (\omega t)$ was used to excite the thermal signals ( $2 \omega$ harmonic temperature and electrical resistance) throughout the wire and the liquid. The measurement procedure mainly consists in sweeping the excitation current frequency, $v=\omega / 2 \pi$, at low values (typically ten values between 0.1 and $1 \mathrm{~Hz}$ ) and measuring the corresponding harmonic variations of the wire temperature (the in-phase and quadrature components), which contain all the needed information about the thermal conductivity of the surrounding liquid [21]. For each of these frequencies, the two temperature component values measured by the DPSD were recorded when they were stabilized to less than $1 \%$ fluctuations. This stabilization procedure usually needs at least fifty periods of measurement accumulation per frequency by the DPSD; this means a measurement time $t_{v, m}$ between $t_{v, m}=500 \mathrm{~s}(v=0.1 \mathrm{~Hz})$ and $t_{v, m}=50 \mathrm{~s}(v=1 \mathrm{~Hz})$ per frequency. The entire process of measuring one thermal conductivity value therefore takes on average about thirty minutes. We repeated at least for times each thermal conductivity measurement and have reported in the present study (see Tables 2 and 3 ) their arithmetic mean values. A maximum relative uncertainty of $5 \%$ was derived from the statistical treatment of our TC measurements.

2.3.2. Validation of the Device. In order to validate the operation of our device, we have firstly measured the room temperature thermal conductivity of usual liquids, such as water and ethanol. The results we obtained were in accordance with the usual values of the thermal conductivity of these liquids, within the limits of the $5 \%$ maximum relative uncertainty mentioned previously. However, as our study focused on the thermal properties of glycerol-based nanofluids, we have refined the calibration of our device by measuring the thermal conductivity $k_{\mathrm{hf}}$ of pure glycerol, as a function of temperature $T$. The results are shown in Table 2 and Figure 4. We have also plotted the linear models obtained from CINDAS values [23] (dashed line) and from our measurements (continuous line). By comparing our results to those obtained in CINDAS, we can conclude that our device gives correct values in the case of glycerol, with an accuracy of around 5\% (a drastic estimation, the worst of our measurements (for $T=320 \mathrm{~K}$ ) being less than $5 \%$ of the corresponding CINDAS value.).

\section{Results}

3.1. Thermal Characterization of Glycerol-Based Nanofluids. The variations of the thermal conductivities $k_{\mathrm{nf}}$ of three different glycerol-based nanofluids have been studied as a function of the solid volume fraction $\phi$ : glycerol $/ \mathrm{SiO}_{2}$, glycer$\mathrm{ol} / \mathrm{CuOCu}_{2} \mathrm{O}$, and glycerol/graphene nanofluids. The samples were all prepared using the same protocol, without any chemical treatment. All the measurements were carried out at the same temperature $T_{i}=298 \mathrm{~K}$, using the device previously described in Section 2.3. The values of the thermal conductivity $k_{\mathrm{nf}}$ measured for each of these nanofluids, as a function of the solid volume fraction $\phi$, are gathered in Table 3.

The variations of the thermal conductivities $k_{\mathrm{nf}}$ of the glycerol-based nanofluids are plotted as a function of the volume fraction $\phi$ in Figures 5, 6, and 7. As can be seen from Figure 5, no particular enhancement of the thermal conductivity has been found in the case of the glycerol/silica nanofluid, with volume fractions of nanoparticles as low as those used here. This was expected, because of the low thermal conductivity of silica $\left(k=1.4 \mathrm{~W} \cdot \mathrm{m}^{-1} \cdot \mathrm{K}^{-1}\right)$. These results are consistent with the results found in the literature. For example, Suleiman et al. concluded that the $\mathrm{SiO}_{2}$ nanoparticles had about $6.1 \%$ higher thermal conductivity enhancement when suspended in ethylene glycol compared with $3.1 \%$ found in glycerol at $30^{\circ} \mathrm{C}$ [24].

The results are however quite different in the case of the nanofluids containing copper oxide nanoparticles or graphene. The best results are obtained in the case of the graphene-based nanofluids, for which a very large enhancement in the thermal conductivity is observed, even at low fractions. This improvement reaches, for example, $224 \%$ in the case of a graphene volume fraction equal to $1 \%$. These very impressive results are due to the high thermal conductivity of graphene and are quite comparable to those obtained with some nanofluids prepared with carbon nanotubes ([2], 200\% increase for a volume fraction as low as $0.35 \%)$ and for graphene-based silicone thermal grease ([25], 120\% enhancement for $1 \%$ of graphene). The thermal conductivities of graphene-water nanofluids with $0.05,0.1$, and $0.15 \%$ volume concentrations and temperatures from 10 to $50^{\circ} \mathrm{C}$ were experimentally studied by Ahammed et al. Enhancements of $19.1 \%$ and $37.2 \%$ were found at $10^{\circ} \mathrm{C}$ and $50^{\circ} \mathrm{C}$, respectively, for the maximum volume concentration of $0.15 \%$ [26].

The thermal conductivity enhancement observed in the case of the intermediate situation of the copper oxidebased nanofluids is also very interesting and can reach a value as high as $121 \%$ in the present case, justifying the interest of such composite systems.

Glycerol-based nanofluids have shown a good improvement in thermal conductivity as proven by several studies in the literature, such as nanofluids containing $\alpha-\mathrm{Al}_{2} \mathrm{O}_{3}$ nanoparticles with three different sizes $(31 \mathrm{~nm}, 55 \mathrm{~nm}$, and $134 \mathrm{~nm}$ ), prepared by a two-step method, at volume 

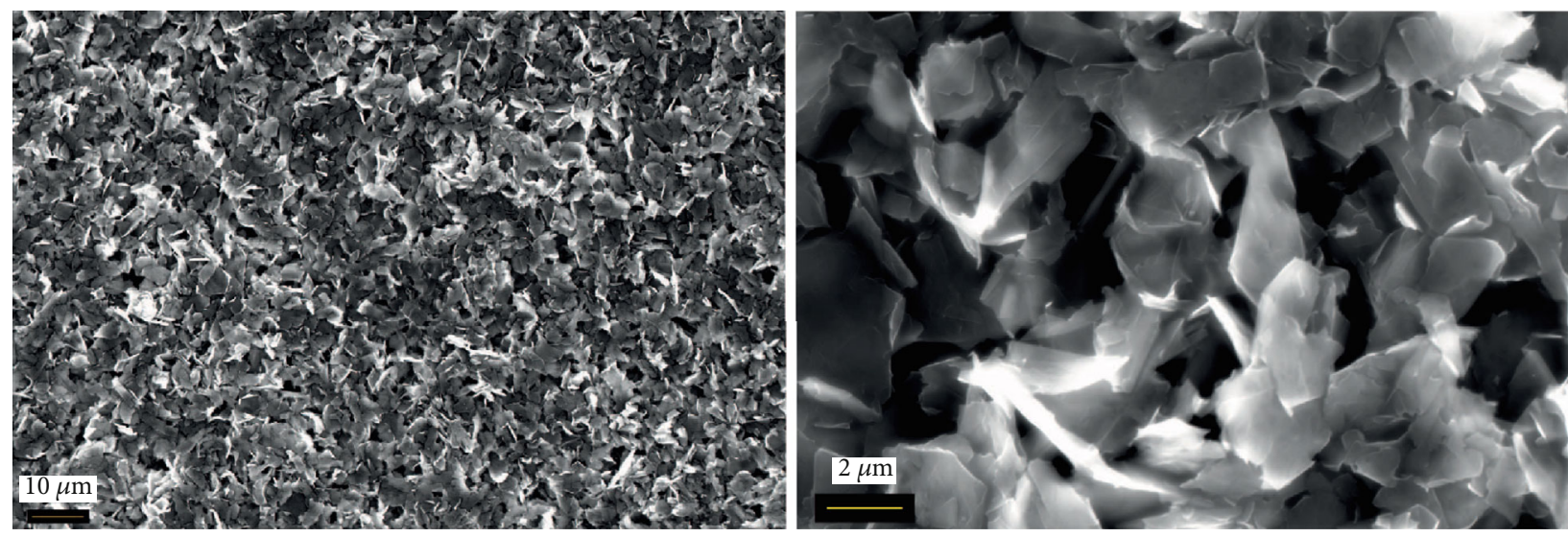

FIGURE 3: SEM characterization of nanofluid glycerol/graphene for a volume fraction $0.25 \%$ of graphene.

TABLE 2: Measurement of the thermal conductivity $k_{\mathrm{hf}}$ of pure glycerol as a function of the temperature. $R^{2}$ is the correlation coefficient. We have indicated the modelling of the data by using a linear law, in the case of our measurements and in the case of CINDAS values.

\begin{tabular}{|c|c|c|c|c|c|c|c|}
\hline$T_{i}(\mathrm{~K})$ & 300 & 305 & 310 & 315 & 320 & 325 & 330 \\
\hline$k_{\mathrm{hf}}\left(\mathrm{W} \cdot \mathrm{m}^{-1} \cdot \mathrm{K}^{-1}\right)$ & 0.284 & 0.285 & 0.285 & 0.287 & 0.286 & 0.287 & 0.288 \\
\hline$R^{2}$ & 0.9994 & 1.0000 & 0.9998 & 0.9991 & 0.9996 & 0.9994 & 0.9999 \\
\hline Model & $k=a T+b$ & $a\left(\mathrm{~W} \cdot \mathrm{m}^{-1} \cdot \mathrm{K}^{-2}\right)$ & $b\left(\mathrm{~W} \cdot \mathrm{m}^{-1} \cdot \mathrm{K}^{-1}\right)$ & & & & \\
\hline Our values & & $1.21 \times 10^{-4}$ & 0.248 & & & & \\
\hline CINDAS values & & $1.294 \times 10^{-4}$ & 0.2464 & & & & \\
\hline
\end{tabular}

fractions ranging from $0.5 \%$ to $4 \%$. The experimental results showed that $\alpha-\mathrm{Al}_{2} \mathrm{O}_{3}-$ glycerol nanofluids have substantially higher thermal conductivity than the base fluid, the maximum enhancement of the relative thermal conductivity being $19.5 \%$ for the case of $31 \mathrm{~nm}$ at $4 \%$ volume fraction [27]. Ntumba et al. have shown that the thermal conductivity ratio increases linearly with the volume fraction of $\mathrm{MgO}$ nanoparticles at a constant temperature and displays a maximum enhancement of $\approx 19 \%$ at $4 \%$ volume fraction of MgO-glycerol [28].

3.2. Analysis and Discussion. There are different elementary models to describe the evolution of the thermal conductivity of suspensions according to the volume fraction $\phi$, among which we consider the Maxwell-Garnett (MG) mixing rule for the case of spherical NPs; it expresses the thermal conductivity ratio of the nanofluid to the base fluid as

$$
\frac{k_{\mathrm{nf}}}{k_{\mathrm{hf}}}=1+\frac{3(\alpha-1) \phi}{\alpha+2-(\alpha-1) \phi} .
$$

In the present case, the thermal contrast $\alpha$ is very high, $\alpha=k_{p} / k_{\mathrm{hf}} \gg 1$; thus, one can approximate (1) as $\phi_{\mathrm{MG}} \approx 1 /(1$ $\left.+3 k_{\mathrm{hf}} / \Delta k\right)$, with $\Delta k=k_{\mathrm{nf}}-k_{\mathrm{hf}}$. In the case of copper oxide nanoparticles suspended in glycerol, we have found that $\Delta k$ $/ k_{\mathrm{hf}}=1.21$ for $\phi_{\text {exp }}=0.64 \%$, while the corresponding value provided by $\mathrm{MG}$ is $\phi_{\mathrm{MG}} \approx 28.7 \%$, i.e., 45 times larger. These results clearly show that the conventional effective medium models are not suitable to describe the improvements of
TABLE 3: Values of the thermal conductivity $k_{\mathrm{nf}}$ (in $\mathrm{W} \cdot \mathrm{m}-{ }^{1} \cdot \mathrm{K}-{ }^{1}$ ), measured for three different glycerol-based nanofluids, as a function of the volume fraction $\varphi$, at room temperature. For each nanofluid, the correlation coefficient $R^{2}$ was greater than 0.99 .

\begin{tabular}{|c|c|c|c|c|}
\hline$\phi(\%)$ & 0.1 & 0.25 & 0.5 & 1.0 \\
\hline Glycerol/SiO ${ }_{2}$ & 0.285 & 0.285 & 0.285 & 0.286 \\
\hline Glycerol/graphene & 0.400 & 0.513 & 0.667 & 0.926 \\
\hline$\phi(\%)$ & 0.078 & 0.16 & 0.32 & 0.64 \\
\hline Glycerol/CuO- $\mathrm{Cu}_{2} \mathrm{O}$ & 0.382 & 0.447 & 0.479 & 0.627 \\
\hline
\end{tabular}

the thermal conductivity observed with the two high thermal contrast glycerol nanofluids studied here.

Another popular model used to determine the thermal conductivity of nanofluids is the Bruggeman model (2). It is valid for spherical particles and considers the interaction between the particles [29].

$$
\begin{aligned}
& \frac{k_{\mathrm{nf}}}{k_{\mathrm{hf}}}=0.25\left[(3 \phi-1) \alpha+(2-3 \phi)+\frac{1}{k_{\mathrm{hf}}} \sqrt{\Delta}\right], \\
& \Delta=\left[(3 \phi-1) k_{p}+(2-3 \phi) k_{\mathrm{hf}}\right]^{2}+8 k_{p} \cdot k_{\mathrm{hf}} .
\end{aligned}
$$

As can be seen from Figures 6 and 7, Bruggeman's law cannot describe the significant increase of the thermal conductivity observed with the glycerol/CuO- $\mathrm{Cu}_{2} \mathrm{O}$ and glycer$\mathrm{ol} /$ graphene nanofluids, even at low volume fractions.

Among the new models proposed to explain the great enhancements of the effective thermal conductivity of the 


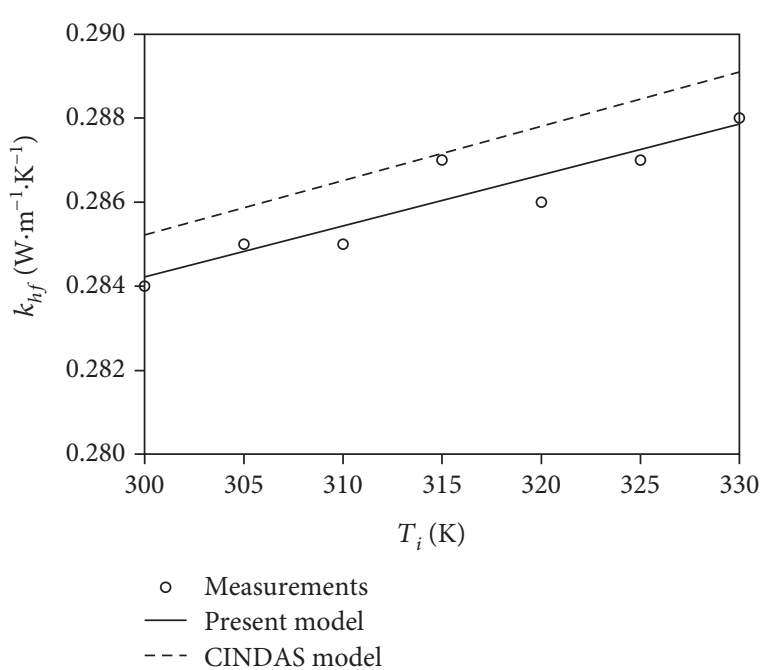

FIGURE 4: Variations of the thermal conductivity $k_{\mathrm{hf}}$ of pure glycerol, as a function of the temperature $T$. The symbols and the continuous line correspond to the measurements made with our device, while the dashed line corresponds to the CINDAS values.

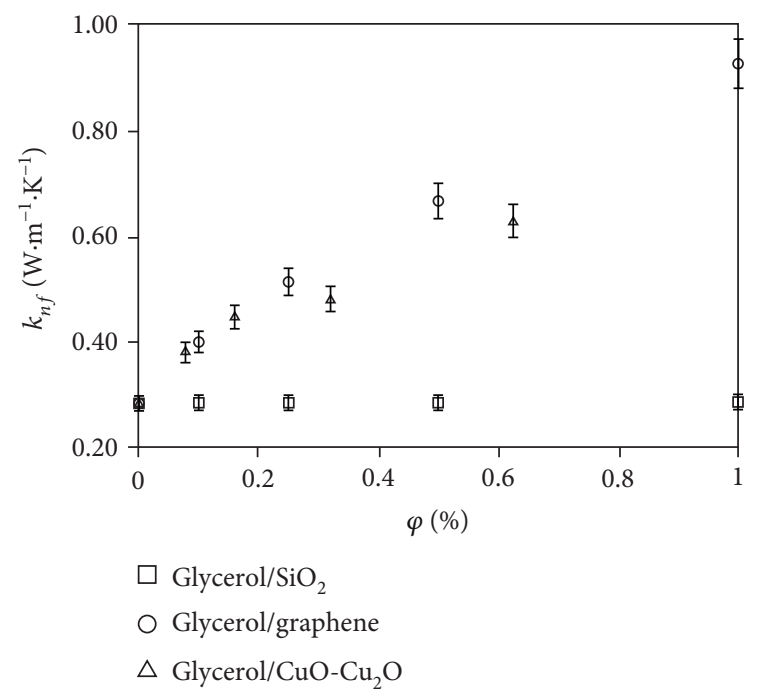

Figure 5: Plot of the thermal conductivity $k_{\mathrm{nf}}$ of glycerol-based nanofluids, as a function of the nanoparticle volume fraction $\phi$, at room temperature.

nanofluids, numerous approaches have underlined the key role played by the Brownian motion of nanoparticles in the microscopic mechanisms of the thermal conductivity enhancements [30]. Most of these models have been introduced in the case of low viscosity base fluids, such as water, and mainly concern spherical nanoparticles. They have been summarized by Chon et al. [31] through the following empirical law:

$$
\frac{k_{\mathrm{nf}}}{k_{\mathrm{hf}}}=1+A \phi^{a}\left(\frac{d_{\mathrm{hf}}}{d_{\mathrm{np}}}\right)^{b}\left(\frac{k_{\mathrm{np}}}{k_{\mathrm{hf}}}\right)^{c} \operatorname{Pr}^{d} \operatorname{Re}^{e},
$$

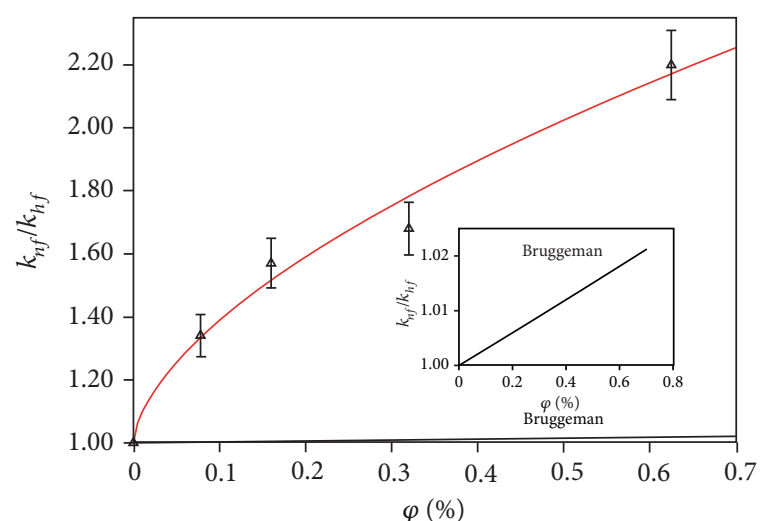

$\triangle$ Glycerol/CuO- $\mathrm{Cu}_{2} \mathrm{O}$

- Modified Chon's law

FIgURE 6: Thermal conductivity enhancement $k_{\mathrm{nf}} / k_{\mathrm{hf}}$ as a function of the nanoparticle volume fraction $\varphi$, in the case of the glycerol/CuO- $\mathrm{Cu}_{2} \mathrm{O}$ nanofluid. The triangular symbols correspond to the measurements, made at the temperature $T_{i}=$ $298 \mathrm{~K}$.

where $A, a, b, c, d$, and $e$ are numbers without dimensions, to be determined for each type of nanofluid; $d_{\mathrm{hf}}=0.547 \mathrm{~nm}$ and $d_{\mathrm{np}}$ are, respectively, the molecular diameter of the base fluid (here the glycerol) and the average diameter of the nanoparticles; $\operatorname{Pr}=c_{p, \mathrm{hf}} \mu_{\mathrm{hf}} / k_{\mathrm{hf}}=12600$ is the Prandtl number of the glycerol at $T=298 \mathrm{~K}$. The Reynolds number based on the Brownian motion of the nanoparticles is given by

$$
\mathrm{Re}=\frac{\rho_{\mathrm{hf}} k_{\mathrm{B}} T}{3 \pi \mu_{\mathrm{hf}}^{2} \ell_{\mathrm{hf}}}
$$

where $k_{\mathrm{B}}$ is the Boltzmann constant; $\rho_{\mathrm{hf}}=1260 \mathrm{~kg} \cdot \mathrm{m}^{-3}$, $\mu_{\mathrm{hf}}=1.5 \mathrm{~Pa} \cdot \mathrm{s}$, and $\ell_{\mathrm{hf}}=0.495 \mathrm{~nm}$ are, respectively, the density, the dynamic viscosity, and the mean-free path of the glycerol at $T=298 \mathrm{~K}$. Chon et al. have determined the following set of values in the case of water $/ \mathrm{Al}_{2} \mathrm{O}_{3}$ nanofluids: $A=64.7, a=0.7460, b=0.3690, c=0.7476, d=0.9955$, and $e=1.2321[31]$.

The same model was used by Corcione et al. $[8,32]$ but with different coefficients.

None of the many models offered in the reviews $[6,7]$ allowed us to describe the results we have obtained with our nanofluids. In a sense, it is relatively logical, because none of the base fluids reported in these reviews is as highly viscous as glycerol.

In the case of the glycerol nanofluids studied here, we have used a modified form of Chon's relation, written as

$$
\frac{k_{\mathrm{nf}}}{k_{\mathrm{hf}}}=1+A^{\prime} \phi^{a}
$$

where $A^{\prime}=A^{\prime}\left(\operatorname{Pr}, R e, d_{\mathrm{hf}} / d_{\mathrm{np}}, k_{\mathrm{np}} / k_{\mathrm{hf}}\right)$ is fluid dependent and is also a function of interfacial resistances. This modified relation has been applied first to the glycerol/CuO- $\mathrm{Cu}_{2} \mathrm{O}$ nanofluid, for which the particles are spherical and of 


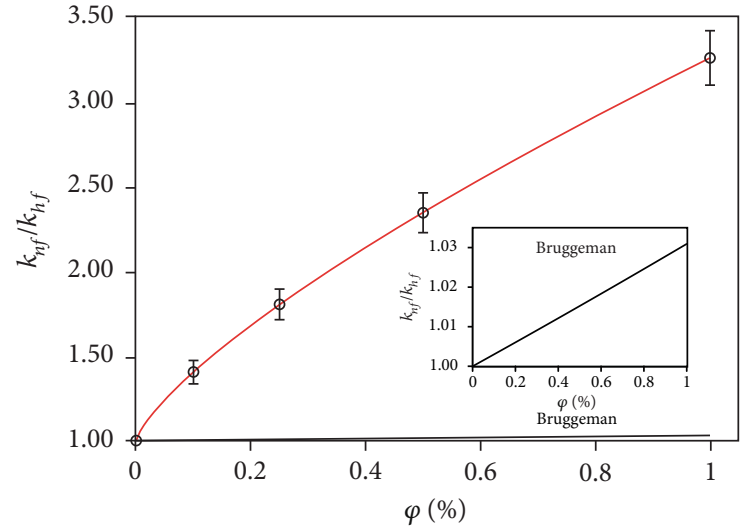

$\bigcirc$ Glycerol/graphene

_ Modified Chon's law

Figure 7: Thermal conductivity enhancement $k_{\mathrm{nf}} / k_{\mathrm{hf}}$ as a function of the nanoparticle volume fraction $\varphi$, in the case of the glycerol/graphene nanofluid. The circular symbols correspond to the measurements, made at the temperature $T_{i}=298 \mathrm{~K}$.

nanometer size, with average values of the nanoparticle thermal conductivity $k_{\mathrm{np}}=50 \mathrm{~W} \cdot \mathrm{m}^{-1} \cdot \mathrm{K}^{-1}$ and diameter $d_{\mathrm{np}}=$ $7 \mathrm{~nm}$. We have found a good agreement between the experimental results and model (6), as shown in Table 4 and Figure 6. The same analysis applies about the modelling of the thermal conductivity of the glycerol/graphene nanofluid. As can be seen in Figure 7, the agreement between the measurements and law (6) is very good in the case of graphene flakes suspended in glycerol. The corresponding value of the exponent $a$, reported in Table 4, is identical to that found by Chon in the case of water-based nanofluids.

The use of a high-viscosity base fluid also allows us to examine the role played by the Brownian motion in the enhancement of the thermal conductivity in the case of glycerol-based nanofluids. According to relation (5), the ratio of the Reynolds numbers associated to the Brownian motion of the spherical NPs, suspended, respectively, in glycerol and in water, can be expressed as

$$
\frac{\operatorname{Re}_{\mathrm{gl}}}{\operatorname{Re}_{\mathrm{wa}}}=\frac{\rho_{\mathrm{gl}}}{\rho_{\mathrm{wa}}} \times \frac{\ell_{\mathrm{wa}}}{\ell_{\mathrm{gl}}} \times\left(\frac{\mu_{\mathrm{wa}}}{\mu_{\mathrm{gl}}}\right)^{2} .
$$

By recalling that the densities of glycerol and water are very close, the same hypothesis being applicable for the mean-free paths, one can write to a very good approximation that $\operatorname{Re}_{\mathrm{gl}} / \operatorname{Re}_{\mathrm{wa}} \approx\left(\mu_{\mathrm{wa}} / \mu_{\mathrm{gl}}\right)^{2}=4 \times 10^{-7}$. Even if the Reynolds number is considerably larger for the water-based nanofluids rather than for the glycerol-based nanofluids, an important enhancement of the thermal conductivity has been observed in the latter case. Therefore, given the very large differences in viscosity between the glycerol and water, it seems difficult, in the case of glycerol-based nanofluids, to attribute a major role to the Brownian motion in the enhancement of the thermal conductivity. Evans et al. have shown that the aggregation of nanoparticles into clusters
TABLE 4: Values of the coefficients $A^{\prime}$ and $a$ corresponding to the relation (6) in the case of thermal conductivity.

\begin{tabular}{lccc}
\hline Nanofluid & $A^{\prime}$ & $a$ & $R^{2}$ \\
\hline Glycerol/CuO-Cu $2 \mathrm{O}$ & 24.88 & 0.602 & 0.9834 \\
Glycerol/graphene & 69.89 & 0.746 & 0.9999 \\
Chon's coefficient & - & 0.746 & - \\
Evan's coefficients & 7.014 & 0.690 & 0.9927 \\
\hline
\end{tabular}

can lead to a significant enhancement of the thermal conductivity, whose value is dictated by the cluster morphology [33]. We have applied relation (6) to the results of their Monte Carlo simulations in the case where the chemical dimension $d_{l}$ of the clusters is equal to the fractal dimension $d_{f}=1.8$, with a thermal contrast $k_{\mathrm{np}} / k_{\mathrm{hf}}=100$ and zero interfacial thermal resistance. We have found a good agreement between their results and our model, with a value of the exponent $a$ close to those found in our experiments (Table 4). We recall that the values of the coefficient $A^{\prime}$ depend, among others, on the nature of the base fluid and on the chemical treatments and thus cannot be compared here.

\section{Conclusion}

In this study, three glycerol-based nanofluids have been thermally characterized at room temperature. The use of silica NPs does not induce any notable enhancement of the thermal conductivity, as expected from the low thermal conductivity of silica. In contrast, a significant increase of the thermal conductivity is observed with copper oxide NPs and even more so with graphene flakes suspended in glycerol, comparable to that obtained with carbon nanotubes in some nanofluids. The latter is ascribed to the high thermal conductivity of graphene.

Effective-medium theories such as the Bruggeman and Maxwell-Garnett laws are totally inadequate to describe these results. However, the dependence of the enhancement factor on the NP volume fraction can be fitted with a modified version of an empirical power law [31] applied to NPs suspended in low-viscosity liquids such as water: $k_{\mathrm{nf}} / k_{\mathrm{hf}}-$ $1+A^{\prime} \phi^{a}$, with values of $a$ very close for the three nanofluids.

In the models considered to describe the enhancement factor in low-viscosity liquids, Brownian motion plays a key role. However, the Reynolds numbers associated with Brownian motion are 6-7 orders of magnitude lower for glycerol-based nanofluids than for water-based ones, so Brownian motion cannot be playing a significant role in the nanofluids studied here. On the other hand, aggregation of NPs into clusters has been shown to lead to significant enhancement of the thermal conductivity [33]. Monte Carlo simulations based on this approach are in good agreement with the empirical power law fitted to our results for glycerol-based nanofluids.

Work is in progress to study the influence of both temperature and NP size on the thermal and mechanical transport properties of glycerol-based nanofluids. 


\section{Data Availability}

I declare that all experimental data can be found in the article. For the bibliographic data used in this manuscript, they can be downloaded via the following links. In case of a data request, please send me an email: lotfimohamed_1999@yahoo.fr.-Bibliographic reference links: [1] https://www.researchgate.net/ publication/236353373_Enhancing_thermal_conductivity_ of_fluids_with_nanoparticles, [2] 10.1063/1.2193161, [3] $10.1063 / 1.1341218$, [4] 10.1155/2014/196074, [5] 10.1016/j.ijheatmasstransfer.2005.07.009, [6] 10.1016/j.ijthermalsci.2006.06.010, [7] 10.1080/01457632.2015.987586, [8] 10.1016/j.enconman.2010.06.072, [9] 10.1016/j.ijheatmasstransfer.2006.02.012, [10] 10.1016/j.matchemphys.2007. 10.029, [11] 10.1115/1.1571080, [12] 10.1063/1.2812699, [13] $10.1063 / 1.2838304$, [14] 10.1088/0957-4484/19/30/305706, [15] 10.1016/j.cej.2013.07.006, [16] 10.1155/2020/2061343, [17] 10.1016/0021-9797(68)90272-5, [18] 10.1016/j.apsusc. 2006.05.023, [19] http://www.theses.fr/2010ORLE2086, [20] 10.1063/1.1141498, [21] https://www.intechopen.com/books/ two-phase-flow-phase-change-and-numerical-modeling/ nanofluids-for-heat-transfer, [22] 10.1016/J.SNA.2011.11.002, [23] https://www.worldcat.org/title/properties-of-inorganicand-organic-fluids/oclc/17107861, [24] https://dx.doi.org/10. 1016/j.icheatmasstransfer.2017.08.001, [25] 10.1016/j.physleta.2013.10.017, [26] https://dx.doi.org/10.1016/j.icheatmasstransfer.2015.11.002, [27] https://dx.doi.org/10.1016/j.icheat -masstransfer.2017.04.001, [28] https://dx.doi.org/10.1080/01457632.2016.1151297, [29] 10.1002/andp.19354160705, [30] 10.1063/1.1756684, [31] 10.1063/1.2093936, [32] $10.1155 / 2016 / 3693249$, and [33] 10.1016/j.ijheatmasstransfer.2007.10.017.

\section{Conflicts of Interest}

The authors declare that they have no conflicts of interest.

\section{References}

[1] U. Choi, Enhancing thermal conductivity of fluids with nanoparticles, vol. 231, Technical Report FED, ASME, New-York, 1995.

[2] Y. Yang, E. Grulke, Z. G. Zhang, and G. Wu, "Thermal and rheological properties of carbon nanotube-in-oil dispersions," Journal of Applied Physics, vol. 99, no. 11, p. 114307, 2006.

[3] J. A. Eastman, S. U. S. Choi, S. Li, W. Yu, and L. J. Thompson, "Anomalously increased effective thermal conductivities of ethylene glycol-based nanofluids containing copper nanoparticles," Applied Physics Letters, vol. 78, no. 6, pp. 718-720, 2001.

[4] Y.-H. Hung and H.-J. Gu, "Multiwalled carbon nanotube nanofluids used for heat dissipation in hybrid green energy systems," Journal of Nanomaterials, vol. 2014, Article ID 196074, 12 pages, 2014.

[5] Y. Ding, H. Alias, D. Wen, and R. A. Williams, "Heat transfer of aqueous suspensions of carbon nanotubes (CNT nanofluids)," International Journal of Heat and Mass Transfer, vol. 49, no. 1-2, pp. 240-250, 2006.
[6] X.-Q. Wang and A. S. Mujumdar, "Heat transfer characteristics of nanofluids: a review," International Journal of Thermal Sciences, vol. 46, no. 1, pp. 1-19, 2007.

[7] H. S. Aybar, M. Sharifpur, M. R. Azizian, M. Mehrabi, and J. P. Meyer, "A review of thermal conductivity models for nanofluids," Heat Transfer Engineering, vol. 36, no. 13, pp. 1085$1110,2015$.

[8] M. Corcione, "Empirical correlating equations for predicting the effective thermal conductivity and dynamic viscosity of nanofluids," Energy Conversion and Management, vol. 52, no. 1, pp. 789-793, 2011.

[9] M.-S. Liu, M. C.-C. Lin, C. Tsai, and C.-C. Wang, "Enhancement of thermal conductivity with $\mathrm{Cu}$ for nanofluids using chemical reduction method," International Journal of Heat and Mass Transfer, vol. 49, no. 17-18, pp. 3028-3033, 2006.

[10] N. Karthikeyan, J. Philip, and B. Raj, "Effect of clustering on the thermal conductivity of nanofluids," Materials Chemistry and Physics, vol. 109, no. 1, pp. 50-55, 2008.

[11] S. K. Das, N. Putra, P. Thiesen, and W. Roetzel, "Temperature dependence of thermal conductivity enhancement for nanofluids," Journal of Heat Transfer, vol. 125, no. 4, pp. 567-574, 2003.

[12] J. Philip, P. D. Shima, and B. Raj, "Enhancement of thermal conductivity in magnetite based nanofluid due to chainlike structures," Applied Physics Letters, vol. 91, no. 20, p. 203108, 2007.

[13] J. Philip, P. Shima, and B. Raj, "Nanofluid with tunable thermal properties," Applied Physics Letters, vol. 92, no. 4, p. 043108, 2008.

[14] J. Philip, P. D. Shima, and B. Raj, "Evidence for enhanced thermal conduction through percolating structures in nanofluids," Nanotechnology, vol. 19, no. 30, p. 305706, 2008.

[15] M. B. Moghaddam, E. K. Goharshadi, M. H. Entezari, and P. Nancarrow, "Preparation, characterization, and rheological properties of graphene-glycerol nanofluids," Chemical Engineering Journal, vol. 231, pp. 365-372, 2013.

[16] S. N. Suhaimi, A. R. A. Rahman, M. F. Md Din, M. Z. Hassan, M. T. Ishak, and M. T. B. Jusoh, "A review on oil-based nanofluid as next-generation insulation for transformer application," Journal of Nanomaterials, vol. 2020, Article ID 2061343, 2020.

[17] W. Stöber, A. Fink, and E. Bohn, "Controlled growth of monodisperse silica spheres in the micron size range," Journal of Colloid and Interface Science, vol. 26, pp. 62-69, 1968.

[18] L. Zhang, R. He, and H. C. Gu, "Oleic acid coating on the monodisperse magnetite nanoparticles," Applied Surface Science., vol. 253, no. 5, pp. 2611-2617, 2006.

[19] A. Hadaoui, Effects of size and concentration on the thermal and rheological properties of nanofluids, $\mathrm{PhD}$ thesis, University of Orléans, France and Cadi Ayyad University of Marrakech, Morocco,, 2010.

[20] D. Cahill, "Thermal conductivity measurement from 30 to 750 $\mathrm{K}$ : the $3 \omega$ method," The Review of Scientific Instruments, vol. 61, no. 2, pp. 802-808, 1990.

[21] R. Heyd, "Nanofluids for heat transfer," Open access peerreviewed chapter, 2011.

[22] R. Heyd, A. Hadaoui, and M.-L. Saboungi, "1D analog behavioral spice model for hot wire sensors in the continuum regime," Sensors and Actuators A: Physical, vol. 174, pp. 915, 2012.

[23] C. Y. Ho, Ed., "Properties of inorganic and organic fluids," in CINDAS Data Series on Material Properties Volume V-1, 
Hemisphere publishing corporation, New York, London, 1988.

[24] S. Akilu, A. T. Baheta, A. A. Minea, and K. V. Sharma, "Rheology and thermal conductivity of non-porous silica $\left(\mathrm{SiO}_{2}\right)$ in viscous glycerol and ethylene glycol based nanofluids," International Communications in Heat and Mass Transfer, vol. 88, pp. 245-253, 2017.

[25] W. Yu, H. Xie, L. Chen, Z. Zhu, J. Zhao, and Z. Zhang, "Graphene based silicone thermal greases," Physics Letters A, vol. 378, no. 3, pp. 207-211, 2014.

[26] N. Ahammed, L. G. Asirvatham, J. Titus, J. R. Bose, and S. Wongwises, "Measurement of thermal conductivity of graphene-water nanofluid at below and above ambient temperatures," International Communications in Heat and Mass Transfer, vol. 70, pp. 66-74, 2016.

[27] M. Sharifpur, N. Tshimanga, J. P. Meyer, and O. Manca, "Experimental investigation and model development for thermal conductivity of $\alpha-\mathrm{Al}_{2} \mathrm{O}_{3}$-glycerol nanofluids," International Communications in Heat and Mass Transfer, vol. 85, pp. 12-22, 2017.

[28] N. Tshimanga, M. Sharifpur, and P. Josua, "Experimental investigation and model development for thermal conductivity of glycerol-MgO nanofluids," Heat Transfer Engineering, vol. 37, no. 18, pp. 1538-1553, 2016.

[29] V. D. A. G. Bruggeman, "Berechnung verschiedener physikalischer konstanten von heterogenen substanzen," Annalen der Physik, vol. 5, no. 24, pp. 636-664, 1935.

[30] S. P. Jang and S. U. S. Choi, "Role of brownian motion in the enhanced thermal conductivity of nanofluids," Applied Physics Letters, vol. 84, no. 21, pp. 4316-4318, 2004.

[31] C. H. Chon, K. D. Kihm, S. P. Lee, and S. U. S. Choi, "Empirical correlation finding the role of temperature and particle size for nanofluid $\left(\mathrm{Al}_{2} \mathrm{O}_{3}\right)$ thermal conductivity enhancement," Applied Physics Letters, vol. 87, no. 15, p. 153107, 2005.

[32] G. Yu, D. Gao, C. Juhui et al., "Experimental research on heat transfer characteristics of $\mathrm{CuO}$ nanofluid in adiabatic condition," Journal of Nanomaterials, vol. 2016, Article ID 3693249, 2016.

[33] W. Evans, R. Prasher, J. Fish, P. Meakin, and P. Phelan, "Effect of aggregation and interfacial thermal resistance on thermal conductivity of nanocomposites and colloidal nanofluids," International Journal of Heat and Mass Transfer, vol. 51, no. 5-6, pp. 1431-1438, 2008. 